\title{
A REDUCED-COMPLEXITY AND ASYMPTOTICALLY EFFICIENT TIME-DELAY ESTIMATOR
}

\author{
Gonzalo Seco;, A. Lee Swindlehurst $\dagger^{\dagger}$ Juan A. Fernández-Rubio*, David Asztély ${ }^{\ddagger}$ \\ ${ }^{*}$ Dept. of Signal Theory \& Communications, Univ. Politècnica de Catalunya, Barcelona, Spain \\ ${ }^{\dagger}$ Dept. of Electrical \& Computer Engineering, Brigham Young University, Provo, Utah \\ ${ }^{\ddagger}$ Research \& Development Dept., Nokia Telecommunications, Kista, Sweden \\ email: gonzalo/juan@gps.tsc.upc.es, swindle@ee.byu.edu, david.astely@nokia.com
}

\begin{abstract}
This paper considers the problem of estimating the time delays of multiple replicas of a known signal received by an array of antennas. Under the assumptions that the noise and co-channel interference (CCI) are spatially colored Gaussian processes and that the spatial signatures are arbitrary, the maximum likelihood (ML) solution to the general time delay estimation problem is derived. The resulting criterion for the delays yields consistent and asymptotically efficient estimates. However, the criterion is highly non-linear, and not conducive to simple minimization procedures. We propose a new cost function that is shown to provide asymptotically efficient delay estimates. We also outline a heuristic way of deriving this cost function. The form of this new estimator lends itself to minimization by the computationally attractive iterative quadratic maximum likelihood (IQML) algorithm. The existence of simple yet accurate initialization schemes based on ESPRIT and identity weightings makes the approach viable for practical implementation.
\end{abstract}

\section{INTRODUCTION}

Synchronization or time delay estimation is a critical aspect of most communications, radar and sonar systems. Accurate frame and symbol synchronization is especially important in time-division multiple access (TDMA) and packetbased systems. Multiuser detectors for code-division multiple access (CDMA) require reliable code timing information for acceptable performance in near-far environments. In addition, achieving precise synchronization is the key to obtain sub-meter accuracies in location estimates with Global Positioning Systems (GPS) receivers.

The performance of single channel timing recovery methods is limited when multipath or CCI is present, such as in many wireless communications systems and surveillance systems plagued by jamming. For this reason, attention has recently shifted to the use of antenna arrays for addressing these problems. The spatial diversity offered by an antenna array can dramatically improve performance in environments with severe interference.

* Work supported by the Catalan and Spanish Governments under grants 1997FI 00755 APDT, TIC98-0412, TIC98-0703, and CIRIT 1998SGR-00081

† Work supported by the U. S. National Science Foundation under Wireless Initiative Grant CCR 99-79452
A number of techniques that exploit antenna arrays for synchronization have been developed, each differing from the others on its assumptions regarding multipath, CCI, signal parameterization and computational load. Some work has focused on determining the direction of arrival (DOA) and time delay of each arrival of a given signal at the array $[1,2,3]$. These methods exploit the full space-time structure of the multipath. Except for some cases that resort to a particular configuration of the antenna array [1], the primary drawback of these approaches is that complicated search procedures are required to estimate the desired parameters. To obtain the DOA estimates, it is necessary to have a calibrated antenna array, and a single arrival at each time delay. Errors in the array calibration are inevitable and, moreover, the latter assumption is unlikely to be valid in a multipath-rich propagation environment. The failure of these two assumptions can lead to significant performance degradation in practical scenarios. In order to overcome these difficulties, an unstructured parameterization of the spatial signatures is used in this paper, as in [4]. Although this leads to an increase in the number of parameters, the model is linear in the additional parameters, and they can be estimated in closed form. Moreover, this assumption offers a good trade-off between model realism and computational complexity.

The technique in [4] has recently been extended in [5] to the blind case where no training data is available. However, the approaches in [4], [5] both assume spatially and temporally white noise, and thus are not suited for situations involving strong CCI. In order to obtain estimators that are robust against the $\mathrm{CCI}$, the unknown and arbitrary spatial correlation of the noise plus interference field has to be estimated. This is only possible if the shape of the desired signal is available, otherwise the model will not be identifiable [6]. The main goal of this paper is to present and analyze an estimator that is asymptotically equivalent to the ML solution and allows the use of computationally appealing minimization algorithms.

\section{DATA MODEL}

We assume that an arbitrary $m$ element array receives $d$ scaled and delayed replicas ${ }^{1}$ of a known signal $s(t)$. The

\footnotetext{
${ }^{1}$ We assume that the number of clusters $d$ is known.
} 
baseband array output is modeled as

$$
\mathbf{y}[n]=\sum_{k=1}^{d} \mathbf{a}_{k} s\left(n T_{s}-\tau_{k}\right)+\mathbf{e}[n]
$$

where $T_{s}$ is the sampling period, $\mathbf{a}_{k}$ and $\tau_{k}$ are the spatial signature and time delay of the $k^{\text {th }}$ arrival, and $\mathbf{e}[n]$ represents additive noise and interference. If $N$ samples are collected, they all may be grouped together into the following equation:

$$
\mathbf{Y}=\left[\begin{array}{llll}
\mathbf{y}[1] & \mathbf{y}[2] & \ldots & \mathbf{y}[N]
\end{array}\right]=\mathbf{A ~ S}(\boldsymbol{\tau})+\mathbf{E}
$$

where $\mathbf{E}$ is formed identically to $\mathbf{Y}$ and

$$
\begin{aligned}
\tau & =\left[\begin{array}{lll}
\tau_{1} & \ldots & \tau_{d}
\end{array}\right]^{T} \quad \mathbf{A}=\left[\begin{array}{lll}
\mathbf{a}_{1} & \ldots & \mathbf{a}_{d}
\end{array}\right] \\
\mathbf{s}[n, \tau] & =\left[\begin{array}{llll}
s\left(n T_{s}-\tau_{1}\right) & \ldots & s\left(n T_{s}-\tau_{d}\right)
\end{array}\right]^{T} \\
\mathbf{S}(\tau) & =\left[\begin{array}{lll}
\mathbf{s}[1, \tau] & \ldots & \mathbf{s}[N, \tau]
\end{array}\right]
\end{aligned}
$$

The term e $[n]$ is modeled as a complex, circularly-symmetric, zero-mean Gaussian process, which is spatially colored with an arbitrary unknown correlation matrix:

$$
\mathcal{E}\left\{\mathbf{e}[n] \mathbf{e}^{*}[m]\right\}=\mathbf{Q} \delta_{n, m}
$$

where $(\cdot)^{*}$ denotes the complex conjugate transpose operation. For simplicity the process is assumed to be temporally white. While such a model for $e[n]$ is clearly only approximate, it captures the most significant effects of the noise and interference, and leads to tractable algorithms. The following additional assumption is needed:

$(A 1): s(t)$ is a band-limited finite-average-power signal, and the sampling satisfies the Nyquist criterion, therefore its autocorrelation function is:

$$
\begin{aligned}
c_{s s}\left(\tau_{k}-\tau_{l}\right) & \triangleq \lim _{T \rightarrow \infty} \frac{1}{T} \int_{T} s\left(t-\tau_{l}\right) s^{*}\left(t-\tau_{k}\right) d t= \\
& =\lim _{N \rightarrow \infty} \frac{1}{N} \sum_{N} s\left(n T_{s}-\tau_{l}\right) s^{*}\left(n T_{s}-\tau_{k}\right)
\end{aligned}
$$

\section{MAXIMUM LIKELIHOOD ESTIMATOR}

Under the model described above and neglecting irrelevant constants, the negative $\log$-likelihood function of $\mathbf{Y}$ is given by

$$
f_{N}(\boldsymbol{\tau}, \mathbf{A}, \mathbf{Q})=\log |\mathbf{Q}|+\operatorname{trace}\left\{\mathbf{C}(\boldsymbol{\tau}, \mathbf{A}) \mathbf{Q}^{-1}\right\},
$$

where

$$
\begin{aligned}
& \mathbf{C}(\boldsymbol{\tau}, \mathbf{A})=\hat{\mathbf{R}}_{y y}-\mathbf{A} \hat{\mathbf{R}}_{y s}^{*}(\boldsymbol{\tau})-\hat{\mathbf{R}}_{y s}(\boldsymbol{\tau}) \mathbf{A}^{*}+\mathbf{A} \hat{\mathbf{R}}_{s s}(\boldsymbol{\tau}) \mathbf{A}^{*} \\
& \hat{\mathbf{R}}_{y y}=\frac{1}{N} \mathbf{Y} \mathbf{Y}^{*} \quad \hat{\mathbf{R}}_{y s}(\boldsymbol{\tau})=\frac{1}{N} \mathbf{Y} \mathbf{S}^{*}(\boldsymbol{\tau}) \\
& \hat{\mathbf{R}}_{s s}(\boldsymbol{\tau})=\frac{1}{N} \mathbf{S}(\boldsymbol{\tau}) \mathbf{S}^{*}(\boldsymbol{\tau})
\end{aligned}
$$

Since $\mathbf{A}$ is taken as an unstructured deterministic matrix, the minimization of (8) may be performed explicitly with respect to $\mathbf{A}$ and $\mathbf{Q}$, whose $\mathrm{ML}$ estimates may be expressed as

$$
\begin{aligned}
& \hat{\mathbf{A}}_{M L}(\boldsymbol{\tau})=\hat{\mathbf{R}}_{y s}(\boldsymbol{\tau}) \hat{\mathbf{R}}_{s s}^{-1}(\boldsymbol{\tau}) \\
& \hat{\mathbf{Q}}_{M L}(\boldsymbol{\tau})=\hat{\mathbf{R}}_{y y}-\hat{\mathbf{R}}_{y s}(\boldsymbol{\tau}) \hat{\mathbf{R}}_{s s}^{-1}(\boldsymbol{\tau}) \hat{\mathbf{R}}_{y s}^{*}(\boldsymbol{\tau})
\end{aligned}
$$

Ignoring parameter independent constants, the resulting criterion for $\tau$ is then

$$
V_{N}(\tau)=\log \left|\mathbf{I}-\mathbf{B}_{N}(\boldsymbol{\tau})\right|
$$

where

$$
\begin{aligned}
& \mathbf{B}_{N}(\boldsymbol{\tau})=\frac{1}{N} \hat{\mathbf{R}}_{y y}^{-\frac{1}{2}} \mathbf{Y} \mathbf{P}_{\mathbf{S}^{*}(\boldsymbol{\tau})} \mathbf{Y}^{*} \hat{\mathbf{R}}_{y y}^{-\frac{1}{2}} \\
& \mathbf{P}_{\mathbf{S}^{*}(\boldsymbol{\tau})}=\mathbf{S}^{*}(\boldsymbol{\tau})\left(\mathbf{S}(\boldsymbol{\tau}) \mathbf{S}^{*}(\boldsymbol{\tau})\right)^{-1} \mathbf{S}(\boldsymbol{\tau})
\end{aligned}
$$

The consistency of the ML time delay estimator follows from the fact that as $N \rightarrow \infty, V_{N}(\tau)$ converges with probability one to its limiting value $V_{\infty}(\tau)$, which is minimized by the true values of the time delays, denoted by the vector $\tau_{0}$. By $(7)$, the limiting value of the cost function is

$$
\begin{aligned}
& V_{\infty}(\tau)=\ln \mid \mathbf{I}- \\
& -\mathbf{R}_{y y}^{-\frac{1}{2}} \mathbf{A C}_{s s}\left(\tau_{0}, \tau\right) \mathbf{C}_{s s}^{-1}(\tau, \tau) \mathbf{C}_{s s}^{*}\left(\tau_{0}, \tau\right) \mathbf{A}^{*} \mathbf{R}_{y y}^{-\frac{1}{2}} \mid
\end{aligned}
$$

where $\mathbf{R}_{y y}$ is the limiting value of $\hat{\mathbf{R}}_{y y}$ and the $k_{z} l$-th element of the matrix $\mathbf{C}_{s s}(\tau, \boldsymbol{\lambda})$ is $c_{s s}\left(\tau_{k}-\lambda_{l}\right)$. The equation (17) satisfies (see [7] for a more detailed proof)

$$
V_{\infty}(\tau) \geq \ln \left|\mathbf{I}-\mathbf{R}_{y y}^{-\frac{1}{2}} \mathbf{A C}_{s s}\left(\tau_{0}, \tau_{0}\right) \mathbf{A}^{*} \mathbf{R}_{y y}^{-\frac{1}{2}}\right|=V_{\infty}\left(\boldsymbol{\tau}_{0}\right)
$$

The equality in (18) holds if and only if

$$
\mathbf{C}_{s s}\left(\tau_{0}, \boldsymbol{\tau}_{0}\right)-\mathbf{C}_{s s}\left(\tau_{0}, \boldsymbol{\tau}\right) \mathbf{C}_{s s}^{-1}(\boldsymbol{\tau}, \boldsymbol{\tau}) \mathbf{C}_{s s}^{*}\left(\boldsymbol{\tau}_{0}, \boldsymbol{\tau}\right)=0
$$

which is only possible for $\tau=\tau_{0}$ if the following nonambiguity condition is fulfilled: The matrix $\mathbf{C}_{s s}(\tilde{\tau}, \tilde{\boldsymbol{\tau}})$ is positive definite for any vector $\tilde{\tau}$ of length $2 d$ whose elements are all distinct. The consistency of $\hat{\mathbf{A}}_{M L}$ and $\hat{\mathbf{Q}}_{M L}$ follows immediately from (12)-(13) and the consistency of $\hat{\tau}_{M L}$. Finally, since the ML estimates of all the parameters are consistent, they will also be asymptotically (large $N$, throughout the paper) efficient.

If the noise is assumed spatially white, the ML cost function that results is

$$
f_{N}^{w}(\boldsymbol{\tau})=-\frac{1}{N} \operatorname{Tr}\left\{\mathbf{Y} \mathbf{P}_{\mathbf{S}^{*}(\boldsymbol{\tau})} \mathbf{Y}^{*}\right\}
$$

which can be minimized using the computationally attractive IQML (or MODE) algorithm (see [4] for details). This algorithm is based on a reparameterization of $\mathbf{P}_{\mathbf{S}^{*}}$ according to the coefficients of a certain polynomial obtained after transforming the data to the frequency domain. Assuming a previous estimate of these coefficients is available, the dependence of $\mathbf{P}_{\mathbf{S}^{*}}$ on some trial coefficients becomes quadratic. The $\mathrm{ML}$ cost function for unknown correlated noise in (14) does not depend linearly on $\mathbf{P}_{\mathbf{S}^{*}}$ due to the determinant operation. Consequently, an IQML-like algorithm cannot be directly applied to (14), which can only be minimized using a burdensome multidimensional search. 


\section{AN ASYMPTOTICALLY EQUIVALENT ESTIMATOR}

In this section, we present a cost function that is asymptotically equivalent to the original ML criterion (14), but that is linear in the signal projection matrix and therefore makes possible the computation of the estimates using an IQML approach. The delay estimates can be computed as the minimizing arguments of

$$
g_{N}\left(\tau, \mathbf{W}_{0}\right)=-\operatorname{Tr}\left\{\mathbf{W}_{0} \mathbf{B}_{N}(\tau)\right\}
$$

where

$$
\mathbf{W}_{0} \triangleq\left(\mathbf{I}-\mathbf{B}_{N}\left(\tau_{0}\right)\right)^{-1}
$$

The estimates obtained from (14) and (21) are asymptotically equivalent (i.e. they only differ in a term of order $\left.o_{p}\left(N^{-1 / 2}\right)\right)$, since the cost functions satisfy the sufficient conditions

$$
\begin{aligned}
& g_{N}^{i}\left(\tau_{0}, \mathbf{W}_{0}\right)=V_{N}^{i}\left(\tau_{0}\right)+o_{p}\left(N^{-1 / 2}\right) \\
& g_{N}^{i j}\left(\boldsymbol{\tau}_{0}, \mathbf{W}_{0}\right)=V_{N}^{i j}\left(\boldsymbol{\tau}_{0}\right)+o_{p}(1)
\end{aligned}
$$

where the superscript $(\cdot)$ denotes the derivative with respect to $\tau_{i}$. A double superscript denotes the corresponding second derivatives. The proof of (24) is based on the fact that $\mathbf{B}_{N}^{i}\left(\tau_{0}\right)=O_{p}\left(N^{-1 / 2}\right)$ [7]. The weighting matrix $\mathbf{W}_{0}$ appearing in the proposed cost function depends on the true value of the delays, and hence is unknown. However, it is well known that we can replace it with a consistent estimate $\hat{\mathbf{W}}$ without affecting the asymptotic properties of the estimates. If $\hat{\tau}$ is a consistent estimate of $\tau_{0}$, then we can construct the practical weighting matrix as

$$
\hat{\mathbf{W}}=\left(\mathbf{I}-\mathbf{B}_{N}(\hat{\boldsymbol{\tau}})\right)^{-1} .
$$

It is worth remarking that the practical cost function admits the following expression:

$$
g_{N}(\boldsymbol{\tau}, \hat{\mathbf{W}})=-\operatorname{Tr}\left\{\hat{\mathbf{Q}}^{-1 / 2} \mathbf{Y} \mathbf{P}_{\mathbf{S}^{*}(\boldsymbol{\tau})} \mathbf{Y}^{*} \hat{\mathbf{Q}}^{-1 / 2}\right\}
$$

where $\hat{\mathbf{Q}}=\hat{\mathbf{R}}_{y y}-\hat{\mathbf{R}}_{y s}(\hat{\boldsymbol{\tau}}) \hat{\mathbf{R}}_{s s}^{-1}(\hat{\boldsymbol{\tau}}) \hat{\mathbf{R}}_{y s}^{*}(\hat{\boldsymbol{\tau}})$ is a consistent estimate of the correlation matrix of the noise. The criterion above resembles the one in the white-noise case $(20)$; the difference is that now the signals are prewhitened using an estimate of the noise correlation. While the function in (26) could have been derived using purely heuristic reasoning, the development followed herein has allowed us to prove the equivalence between (26) and the original criterion (14), which would have been difficult to do from a simple inspection of those cost functions.

The use of (26) is advantageous as long as the initial consistent estimate of the delays can also be computed easily. Indeed, this is possible, and $\hat{\tau}$ can be obtained as the minimizing argument of $g_{N}(\tau, \mathbf{I})$, in which the unknown weighting matrix is replaced by the identity, which amounts to prewhitening the signals according to the total correlation matrix $\hat{\mathbf{R}}_{y y}$ instead of the correlation of the noise. Note that the criterion $f_{N}^{w}(\tau)$ also provides consistent estimates. Nevertheless, as illustrated in the simulations, its performance is very poor in the presence of interference.

\section{HEURISTIC DERIVATION}

In the previous section, we have directly put forward the new cost function $g_{N}(\tau, \hat{\mathbf{W}})$ and determined its asymptotic behavior. In this section, one of the three heuristic approaches presented in [7] to derive that function is described. Note that this approach is rather general and may thus be of interest for other problems as well.

Since the eigenvalues of $\mathbf{B}_{N}(\tau)$ satisfy $0 \leq \lambda_{i}<1$, the original ML criterion admits the following series expansion

$$
V_{N}(\boldsymbol{\tau})=-\sum_{k=1}^{\infty} \frac{1}{k} \operatorname{Tr}\left\{\mathbf{B}_{N}^{k}(\boldsymbol{\tau})\right\}
$$

The function $g_{N}(\tau, \mathbf{I})$, which we have proposed to use in obtaining the initial consistent estimates, is the first term of this expansion. Unlike many other estimation problems (see e.g., [6]), the first-order term is not asymptotically equivalent to the original function because $\lim _{N \rightarrow \infty} \mathbf{B}_{N}\left(\tau_{0}\right)=$ $\mathbf{I}-\mathbf{R}_{y y}^{-1 / 2} \mathbf{Q} \mathbf{R}_{y y}^{-1 / 2}$ is not equal to zero. In order to maintain not only consistency but also asymptotic efficiency, all of the terms in the expansion (27) must be kept. Since the second and higher-order terms are the ones that introduce the undesirable non-linear dependence on the matrix $\mathbf{P}_{\mathbf{S}^{*}(\tau)}$, we decide to approximate them. The approximation is not done directly over $V_{N}(\tau)$, but over its derivative. If we differentiate (27) and replace $\mathbf{B}_{N}(\tau)$ by $\mathbf{B}_{N}(\hat{\boldsymbol{\tau}})$ in all the second and higher-order terms (this is justified since $\left.\mathbf{B}_{N}^{i}\left(\tau_{0}\right)=O_{p}\left(N^{-1 / 2}\right)\right)$, it results that

$$
\begin{aligned}
V_{N}^{i}(\boldsymbol{\tau}) & \simeq-\operatorname{Tr}\left\{\mathbf{B}_{N}^{i}(\boldsymbol{\tau})\left(\mathbf{I}+\mathbf{B}_{N}(\hat{\boldsymbol{\tau}})+\mathbf{B}_{N}^{2}(\hat{\boldsymbol{\tau}})+\cdots\right)\right\} \\
& =-\operatorname{Tr}\left\{\left(\mathbf{I}-\mathbf{B}_{N}(\hat{\boldsymbol{\tau}})\right)^{-1} \mathbf{B}_{N}^{i}(\boldsymbol{\tau})\right\}
\end{aligned}
$$

Thus we retrieve the new criterion presented in the Section 4 since the value of $\boldsymbol{\tau}$ that minimizes $g(\tau, \hat{\mathbf{W}})$ also nulls (28).

\section{SIMULATION RESULTS}

We analyze the performance of the estimators proposed in this paper, and compare it with the Cramér-Rao Bound (CRB). The cost function $g(\tau, \hat{\mathbf{W}})$ is minimized using IQML, while the initial delay estimates are obtained by applying the ESPRIT algorithm to $g(\tau, \mathbf{I})$. The use of both IQML and ESPRIT in the white-noise case is detailed in [4]. Given the similarity between the two functions above and $f_{N}^{w}(\tau)$, the extension of these two algorithms to the correlated-noise case is easy. The iterations of the IQML algorithm are coupled with those related to the computation of the consistent estimate $\hat{\mathbf{W}}$. That is, we have chosen to update this weight matrix at every iteration of IQML.

We concentrate on a scenario where $d=2$ delayed versions of a known signal are received by a uniform linear array with antennas spaced $0.5 \lambda$ apart. This known signal is a concatenation of $K$ truncated and sampled Nyquist squared root raised cosine pulses. Each pulse has a bandwidth equal to $(1+\alpha) / 2 T_{c}$, is truncated to the interval $\left[-3 T_{c}, 3 T_{c}\right]$, and the sampling period is $T_{c} / 2$. The roll-off factor is set equal to $\alpha=0.2$. The noise plus interference field in which the array operates consists of: i) spatially and temporally white Gaussian noise, and $i i$ ) a temporally 
white Gaussian interference at DOA $-30^{\circ}$ relative to the array broadside. The remaining scenario parameters, except when one of them is varied, are as follows: $m=6$ antennas; $K=4$ pulses; delays of the two signals equal to 0 and $0.4 T_{c}$; DOAs of those signals: $0^{\circ}, 10^{\circ}$; Signal to Noise Ratio (SNR) of the first signal: $16 \mathrm{~dB}$; Signal to Interference Ratio (SIR) of the first signal: $-3 \mathrm{~dB}$; the second signal is attenuated $3 \mathrm{~dB}$ with respect to the first, and they are in phase at the first sensor.

The finite-sample and asymptotic performance of the different estimators is illustrated in Fig.1. As predicted by the theoretical study, the RMSE of the proposed cost function in (26) tends to the CRB as the number of samples increases. Indeed, the consistent estimator $g_{N}(\tau, I)$ does not attain the $\mathrm{CRB}$, but it is robust against the presence of the directional interferer, as shown in Fig.2. This occurs because this estimator takes into account the spatial correlation of the noise field, though not in an optimal way. On the contrary, the method designed for the white-noise case undergoes a severe degradation with respect to the other two estimators and is not appropriate for scenarios with strong or even moderate CCI (see Fig.2). When the DOA separation of the signals is smaller than the beamwidth of the sensor array, the CRB increases as the DOA separation decreases. Also in this case, the performance of ESPRIT is impaired because the matrix $\mathbf{A}$ tends to be rank deficient. However, the RMSE of the proposed method is always very close to the CRB, even though it is initialized with ESPRIT.

\section{CONCLUSIONS}

The ML solution to the general time delay estimation problem in unknown spatially colored noise has been derived. It has been shown that the resulting criterion is consistent and asymptotically efficient, but it results in a non-linear multidimensional minimization problem. We have developed a new estimator that is asymptotically equivalent to the ML criterion and allows the use of the attractive IQML algorithm. A simple initialization method with excellent performance has also been proposed.

\section{REFERENCES}

[1] A.-J. van der Veen, M. C. Vanderveen, and A. Paulraj, "Joint Angle and Delay Estimation Using ShiftInvariance Techniques," IEEE Trans. SP, vol. 46, pp. 405-418, Feb. 1998.

[2] M. Cedervall and A. Paulraj, "Joint Channel and SpaceTime Parameter Estimation," in Proc. 30th Asilomar Conf. on Signals, Systems, and Computers, pp. 375$379,1996$.

[3] M. Wax and A. Leshem, "Joint Estimation of Time Delays and Directions of Arrival of Multiple Reflections of a Known Signal," IEEE Trans. on SP, vol. 45, pp. 24772484, Oct. 1997.

[4] A. L. Swindlehurst, "Time Delay and Spatial Signature Estimation Using Known Asynchronous Signals," IEEE Trans. SP, vol. 46, pp. 449-462, Feb. 1998.

[5] A. Swindlehurst and J. Gunther, "Methods for Blind Equalization and Resolution of Overlapping Echoes of

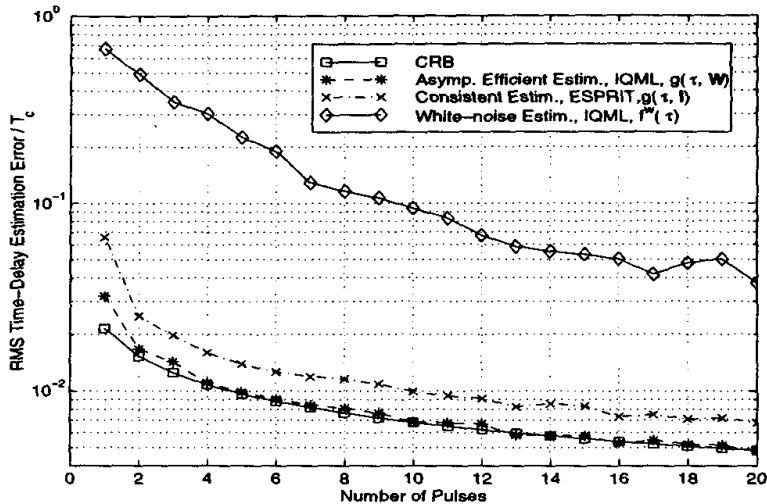

Figure 1: RMSE versus the number of training pulses.

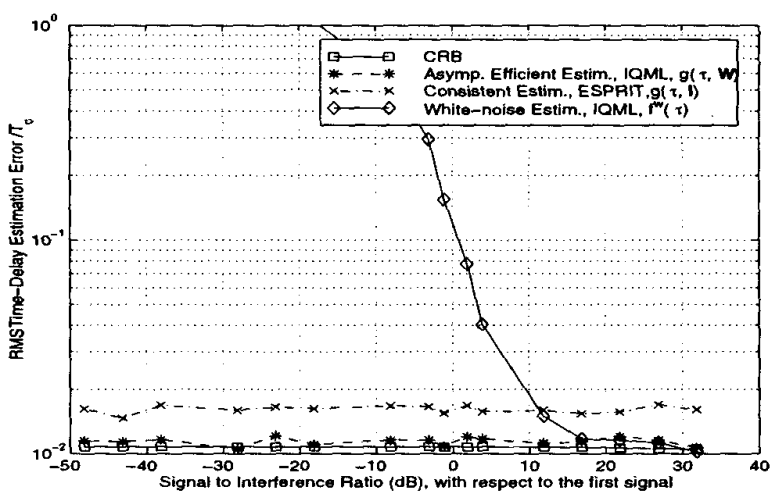

Figure 2: RMSE versus the interference power.

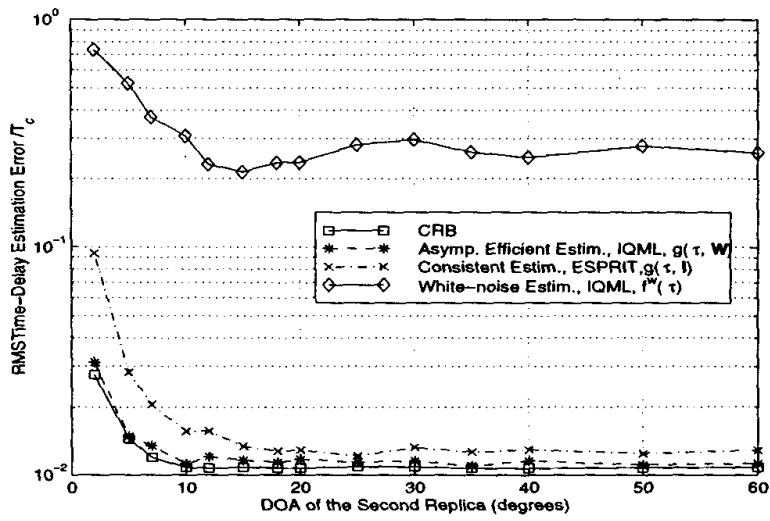

Figure 3: RMSE versus DOA separation.

Unknown Shape," IEEE Trans. SP, vol. 47, pp. 12451254, May 1999.

[6] D. Zheng, J. Li, S. Miller, and E. Ström, "An Efficient Code-Timing Estimator for DS-CDMA Signals," IEEE Trans. SP, vol. 45, pp. 82-89, Jan. 1997.

[7] G. Seco, A. Swindlehurst, and D.Asztély, "Exploiting Antenna Arrays for Synchronization." In preparation. 